\title{
The spin pulse of the intermediate polar V1062 Tauri
}

\author{
C. Hellier ${ }^{1}$, A. P. Beardmore ${ }^{1}$, and K. Mukai ${ }^{2}$ \\ 1 Astrophysics Group, School of Chemistry and Physics, Keele University, Staffordshire ST5 5BG, UK \\ 2 Laboratory for High Energy Physics, NASA/GSFC, Code 662, Greenbelt, MD 20771, \\ and Universities Space Research Association, USA
}

Received 18 February 2002 / Accepted 22 April 2002

\begin{abstract}
We combine ASCA and RXTE data of V1062 Tau to confirm the presence of a 62-min X-ray pulsation. We show that the pulsation is caused largely by the variation of dense partial absorption, in keeping with current models of accretion onto magnetic white dwarfs. Further parametrisation of the spin pulse is, however, hampered by ambiguities in the models.
\end{abstract}

Key words. accretion, accretion disks - stars: individual: V1062 Tau - novae, cataclysmic variables binaries: close $-\mathrm{X}$-rays: stars.

\section{Introduction}

The intermediate polar (IP) class of cataclysmic variable is characterised by prominent X-ray pulsations resulting from accretion onto magnetic white dwarfs (see Patterson 1994 for a review). Several studies have attempted to use spectral analysis of X-ray data to deduce the cause of the pulsations (e.g. Rosen et al. 1998; Hellier et al. 1991; de Martino et al. 2001). In particular, some studies have argued that the pulses are caused by accretion regions moving over the white-dwarf limb for parts of the white-dwarf spin cycle (e.g. Singh \& Swank 1993; Allan et al. 1998), whereas other studies have suggested that the prime cause is absorption occurring when parts of the accretion flow cross the line of sight (e.g. Rosen et al. 1998; Hellier et al. 1991). In general, both effects may be important.

In probing further, we have found that spectral analysis often gives ambiguous results. Phase-varying absorption must be partial, and of several different densities, while a disappearance over the limb must take into account the temperature-stratification of the accretion column. The lowest, coolest regions disappear first, which can mimic the spectral signature of partial absorption. In at least one star, V1025 Cen, we found that ASCA data alone could not distinguish between different complex models (Hellier et al. 1998). We have thus obtained simultaneous $A S C A$ and RXTE data of an IP, aiming to combine the spectral resolution of $A S C A$ with the harder response and higher throughput of RXTE to reduce the ambiguities. As

Send offprint requests to: C. Hellier,

e-mail: ch@astro.keele.ac.uk a target we chose V1062 Tau, with the additional aim of confirming its membership of the IP class, given that the X-ray spin pulse was previously known from only 6 cycles of data (Remillard et al. 1994). With both a spin period (62 min) and an orbital period $(10 \mathrm{~h})$ that are unusually long, V1062 Tau marks one extreme of the $P_{\text {spin }}-P_{\text {orb }}$ plane (e.g. Hellier 1999), though it fits the $P_{\text {spin }} \approx 0.1 P_{\text {orb }}$ rule-of-thumb that many IPs obey.

\section{The data}

We observed V1062 Tau between 1998 February 16 06:38 UT and 1998 February 18 13:25 UT in a simultaneous observation using the RXTE and ASCA satellites (see Bradt et al. 1993 and Tanaka et al. 1994 respectively). $A S C A$ recorded $60 \mathrm{ks}$ of good data in the energy range $0.6-10 \mathrm{keV}$. The count rate was $0.3 \mathrm{~s}^{-1}$ in each of the 4 instruments (SIS0, SIS1, GIS2, GIS3). RXTE recorded $51 \mathrm{ks}$ of data with the PCA instrument with all 5 PCUs working. To maximise the signal-to-noise we used only the top xenon layer of PCA data, which after background subtraction using PCABACKEST v2.0c gave an average of 19 counts s ${ }^{-1}$ in 5 PCUs in the range $2-15 \mathrm{keV}$, a similar flux level to that reported by Remillard et al. (1994).

In Fig. 1 we show the combined $1-15 \mathrm{keV}$ RXTE and ASCA lightcurve of V1062 Tau. This results from normalizing the data from the different instruments to the same count rate (a factor 15.7 between ASCA and RXTE) and then merging the lightcurves. Since the instruments have different spectral responses this is a dubious procedure, but for period searching the overriding consideration is to maximise the data coverage. Figure 2 shows the Fourier transform of the lightcurve. There is a clear periodicity 


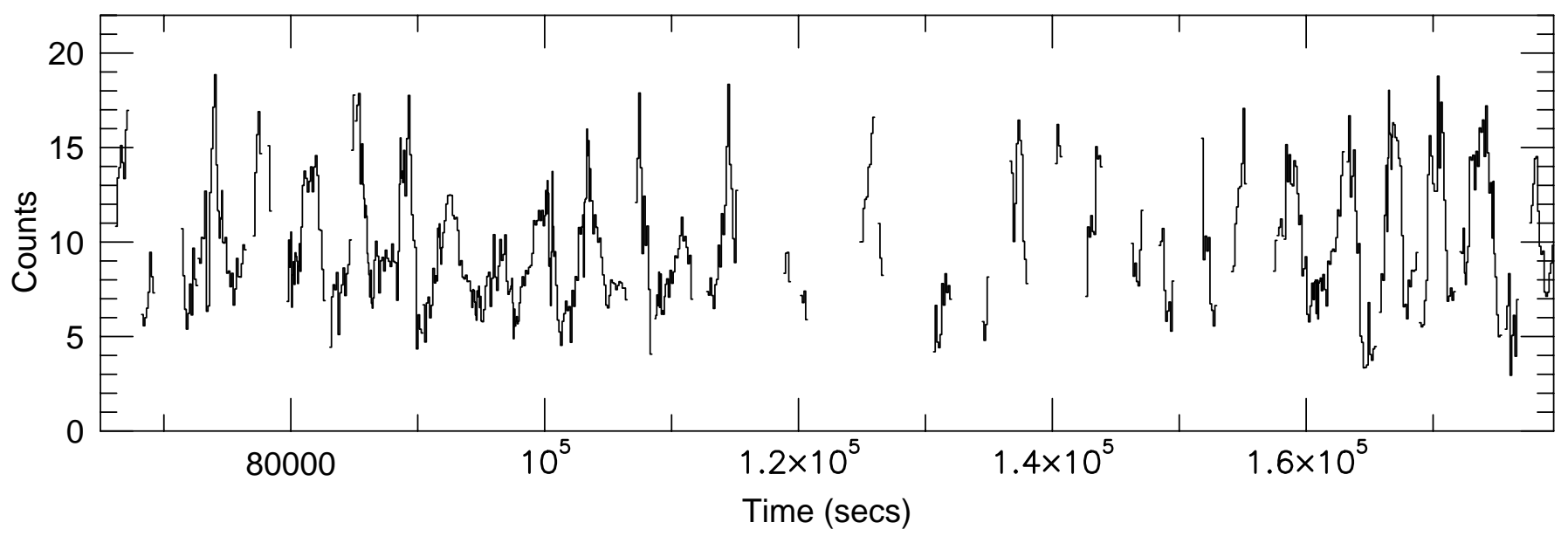

Fig. 1. Part of the combined ASCA and RXTE 1-15-keV lightcurve of V1062 Tau, in 128-s bins.

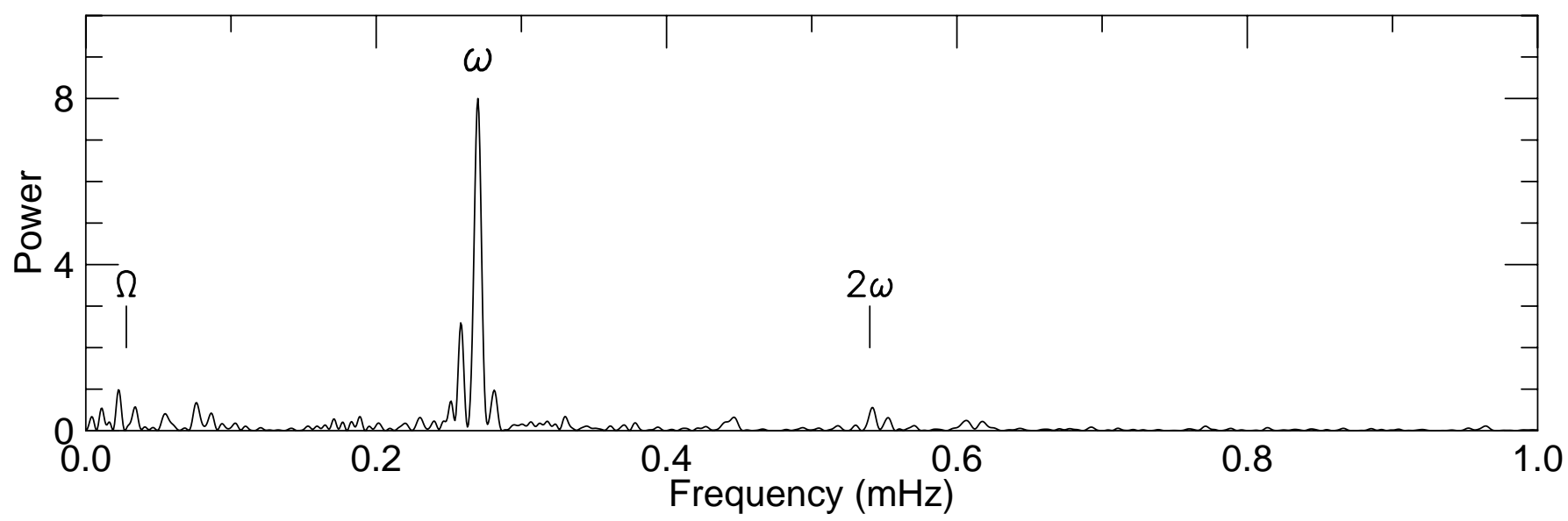

Fig. 2. The Fourier transform of the combined ASCA and RXTE 1-15-keV lightcurve of V1062 Tau. The spin and orbital frequencies are denoted $\omega$ and $\Omega$ respectively.

at $3704 \pm 8 \mathrm{~s}$, consistent with the spin period detected by Remillard et al. (1994), thus confirming that V1062 Tau is an IP. There is also power at the first harmonic of the spin period $(2 \omega)$. The roughly sinusoidal pulse profile is shown in Fig. 3.

There is no significant power at the 10-hr orbital period reported by Remillard et al. (1994), nor any at the beat period between the spin and orbital cycles. We can thus assume that the accretion stream does not connect directly to field lines and that V1062 Tau is a purely discfed accretor (see, e.g., Hellier 1991), which is as expected given the long orbital period.

\section{Spectral analysis}

For phase-resolved analysis we divided the data into four bins, with phases $0.867-0.180$ (maximum); 0.180-0.270 (fall); 0.270-0.695 (minimum); and 0.695-0.867 (rise). We then added the ASCA SIS0 and SIS1 spectra, and also the GIS2 and GIS3 spectra, so obtaining three spectra per phase bin (SIS, GIS and PCA) for simultaneous analysis in XSPEC. We encountered two problems. First, the spectrum of the source showed secular changes

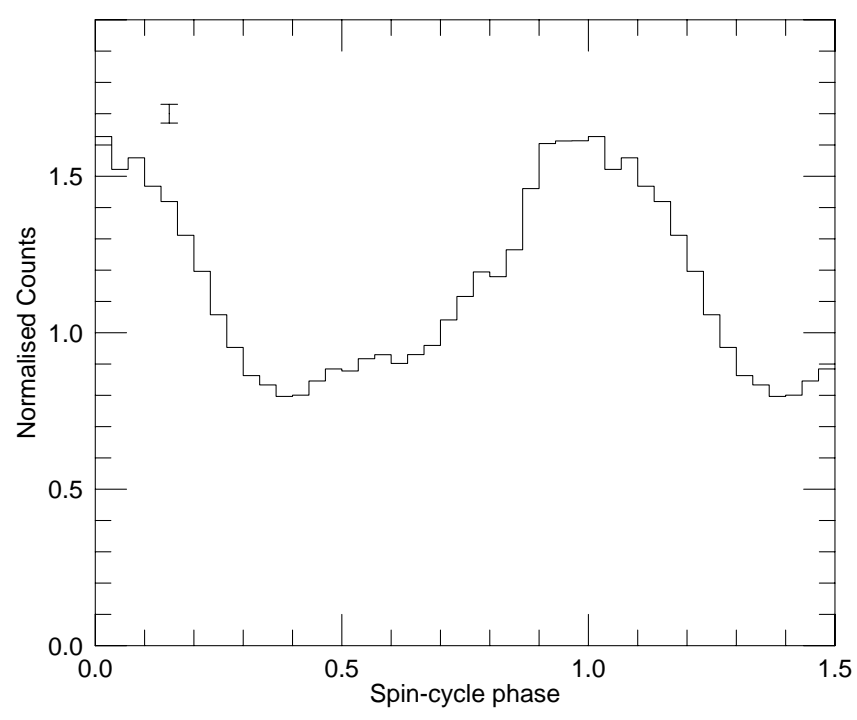

Fig. 3. The combined $A S C A$ and RXTE lightcurve folded on the 3704 -s spin cycle. Phase zero corresponds to TDB(JD) 2450860.77986 .

after 1998 February 18 02:00 UT. The source first became $30 \%$ brighter, then, four hours after the brightening, the 
spectrum became more absorbed, with the hardness ratio $((6-10) /(2-6) \mathrm{keV}$ in the ASCA data) increasing from 0.25 to 0.50 . Thus, for phase-resolved analysis, we excluded the data after 02:00 UT.

Secondly, we found discrepancies in the normalisations between the SIS, GIS and PCA spectra, with the PCA spectra being systematically $\sim 20 \%$ brighter than the GIS spectra, which were $\sim 10 \%$ brighter than the SIS spectra. Some part of this is accounted for by the different sampling of the lightcurves, caused by the different spacecraft orbits; we estimate this to be a $\sim 10 \%$ effect. The remainder is probably the result of calibration uncertainties; note that a $20 \%$ discrepancy between RXTE and ASCA has been reported by Yaqoob (2000). To overcome the problem we fitted a simple power-law model to both $A S C A$ and $R X T E$ spectra, using only the $2-6 \mathrm{keV}$ region of overlap; we then adjusted the exposure times of the spectra until the fitted normalisations matched. In the following modelling we used the data ranges $0.8-9 \mathrm{keV}$ (SIS), $0.8-11 \mathrm{keV}$ (GIS) and 2-25 keV (PCA).

\subsection{The basic model}

The accretion column of an IP is expected to consist of $\sim 30 \mathrm{keV}$ plasma at the accretion shock, cooling to $<1 \mathrm{keV}$ nearer the white dwarf, and becoming optically thick at some point. The temperature and density as a function of height are approximated by the Aizu model (Aizu 1973). Ramsay (2000) has fitted the RXTE spectrum of V1062 Tau using an Aizu distribution of temperatures in a paper aimed at deducing the white-dwarf masses in the class of IPs. Here, we are investigating the spectral changes over spin phase, and so use a simpler, but adequate, parametrisation involving a two-component plasma with temperatures of typically 1 and $30 \mathrm{keV}$.

The multi-temperature spectrum is modified by absorption and scattering by the infalling accretion flow. We found that we needed both simple absorption and partialcovering absorption to reproduce the spectrum. We also obtained a better fit by introducing an iron edge fixed at $7 \mathrm{keV}$, as would arise from strong ionized absorption (fits without this edge typically had $\chi_{\nu}^{2}$ poorer by 0.1$)$. Lastly, we added a narrow line fixed at $6.4 \mathrm{keV}$ to model the iron fluorescence arising from reflection, though for simplicity we did not include a reflection continuum.

When fitting GIS, SIS and PCA spectra simultaneously in 4 phase bins, the above model gave fits with $\chi_{\nu}^{2}$ typically $1.1-1.2$. If the model was optimised for each individual spectrum the resulting $\chi_{\nu}^{2}$ dropped to $\approx 1$, suggesting that calibration uncertainties are largely responsible for the higher $\chi_{\nu}^{2}$ in the simultaneous fits.

\subsection{A variable-absorption model}

We present in Table 1 a model which varies the absorption to reproduce the spin pulse, fixing the temperatures and normalisations over spin phase. The fitted temperatures
Table 1. The variable-absorption model. The basic model is a $0.16-\mathrm{keV}$ MEKAL (normalisation 0.74) plus a $35-\mathrm{keV}$ MEKAL (normalisation 0.023), a simple absorbing column of $1.7 \times$ $10^{22} \mathrm{~cm}^{-2}$, a $7.0-\mathrm{keV}$ edge of $\tau=0.12$, and a $6.4-\mathrm{keV}$ line. The columns and covering fractions $(f)$ of the partial absorption are tabulated. The fit to the SIS+GIS+PCA data simultaneously in 4 phase bins gave $\chi_{\nu}^{2}=1.16(\nu=1548)$.

\begin{tabular}{lcccc}
\hline \hline Partial absorbers & Max & Fall & Min & Rise \\
\hline$n \mathrm{H}\left(\times 10^{22} \mathrm{~cm}^{-2}\right)$ & - & 61 & 8.6 & 19 \\
$f$ & - & 0.40 & 0.69 & 0.33 \\
$n \mathrm{H}\left(\times 10^{22} \mathrm{~cm}^{-2}\right)$ & - & - & $>10^{3}$ & - \\
$f$ & - & - & 0.13 & - \\
\hline
\end{tabular}

(0.16 and $35 \mathrm{keV})$ are ill-constrained and, since we are not fitting with a full column model, we treat them as indicative only (see Ramsay 2000 for a physically motivated analysis of the spectrum). Also fixed over spin phase are a simple absorbing column of $1.7 \times 10^{22} \mathrm{~cm}^{-2}$, a $7.0-\mathrm{keV}$ edge of optical depth 0.12 , and a $6.4-\mathrm{keV}$ line.

From here we reproduce the spin pulse by changing the column and covering fraction of one partial-covering absorber. Further, to model the spin-minimum bin we need an additional dense absorber with column $>10^{25} \mathrm{~cm}^{-2}$ covering 0.13 of the source. Of course, given the high column, this is equivalent to a $13 \%$ flux reduction.

Thus we can model the phase-resolved ASCA $+R X T E$ spectra using 7 phase-independent parameters, a further 2 absorption parameters per phase bin, and the normalisation reduction at spin minimum. The overall $\chi_{\nu}^{2}$ was 1.16 . Adding further parameters, such as allowing the simple column and the optical depth of the $7.0-\mathrm{keV}$ edge to vary over spin phase, reduces the $\chi_{\nu}^{2}$ to 1.09 , but makes little difference to the model as a whole.

Modelling the spin pulse with phase-variable absorption can be interpreted in the standard accretion-curtain model for IPs (Rosen et al. 1998; Hellier et al. 1991; Kim \& Beuermann et al. 1995; Hellier et al. 1996) in which the upper pole points towards the observer at pulse minimum. The accretion flow then obscures the line of sight to the accretion regions, causing the absorption. In this model a partial absorber can be interpreted as either partial covering of a spatially extended emitting region, or the time-average of varying absorption. The $13 \%$ flux reduction at spin minimum then implies that part of the curtain is dense enough to be opaque, and that this covers the emitting region at spin minimum, either partially or for a fraction of the time. The column of $>10^{25} \mathrm{~cm}^{-2}$ is consistent with looking down an accretion column end-on (see, e.g., the column estimates in Hellier et al. 1996).

Alternatively, the disappearance of the lower pole over the white-dwarf limb at spin minimum might contribute to the flux reduction. In the simplest model of a symmetric dipole and zero shock height, the appearance of one pole compensates for the disappearance of the other. However, an asymmetry or a non-zero shock height allows both poles to be visible when the upper pole points away 
Table 2. The variable-normalisation model. It consists of a 1.5-keV MEKAL plus a 34-keV MEKAL, a simple absorbing column of $1.5 \times 10^{22} \mathrm{~cm}^{-2}$, another $10.1 \times 10^{22} \mathrm{~cm}^{-2}$ column covering $71 \%$ of the source, a $7.0-\mathrm{keV}$ edge of $\tau=0.18$, and a $6.4-\mathrm{keV}$ line. The normalisations of the two plasma components are tabulated. The fit gave $\chi_{\nu}^{2}=1.24(\nu=1550)$.

\begin{tabular}{lcccc}
\hline \hline & Max & Fall & Min & Rise \\
\hline 34-keV norm $\left(\times 10^{-2}\right)$ & 2.56 & 1.95 & 2.15 & 2.36 \\
$1.5-\mathrm{keV}$ norm $\left(\times 10^{-2}\right)$ & 3.47 & 1.39 & 0.0 & 1.86 \\
\hline
\end{tabular}

from us (spin maximum), with only one pole visible when the upper pole points towards us (e.g., Mukai 1999).

\subsection{A variable-normalisation model}

As an alternative to varying the absorption, we have explored whether the spin pulse can be modelled by changing only the normalisation of the spectral components, as would occur if parts of the accretion region were passing over the white-dwarf limb. Even if the absorption is not phase-varying, a partial-covering absorber of $\sim 10^{23} \mathrm{~cm}^{-2}$ is still required to model the spectrum: multi-temperature models with no absorption, or with much lower absorption, give $\chi_{\nu}^{2}>2.4$. We thus use the same basic model as above, a two-temperature plasma with partial-covering absorbers, an iron edge, and an iron fluorescence line.

From here, the spin pulse is reproduced by modulating the low-temperature plasma by $100 \%$ while the hightemperature plasma is modulated by $30 \%$ (see Table 2 ). This model gives $\chi_{\nu}^{2}=1.24$, somewhat worse than the 1.16 obtained with the variable-absorption model.

We can ask, further, whether this model makes physical sense. For instance, it is unlikely that a $10^{23} \mathrm{~cm}^{-2}$ absorber covering $71 \%$ of the source would not change over phase; such absorption can only come from the magnetically trapped accretion flow, and this would wheel round on the spin cycle, coming into and out of the line of sight. Second, producing a spin-pulse by changing the normalisations requires that parts of the accretion regions be passing over the white-dwarf limb at all spin phases (see Table 2), and this requires large, extended accretion regions, comparable in size to the white-dwarf surface. However, this conflicts with the fact that the low-temperature component is $100 \%$ modulated, since, even if there is some asymmetry between the upper and lower poles, it is inconsistent that the accretion regions are (1) comparable to the white dwarf in size, (2) disappear completely over the whitedwarf limb, and (3) are not replaced by the appearance of the opposite accretion region. Further, the requirement for large accretion regions conflicts with the finding that they cover only $<0.002$ of the white-dwarf surface in XY Ari (Hellier 1997). For all of the above reasons, we consider that reproducing the spin pulse by varying normalisations alone is not viable.

Of course, we cannot rule out the possibility that occultation plays a secondary role, since allowing both absorption and occultation to vary over spin phase produces a wide range of acceptable but non-unique fits.

\section{Discussion}

To model the X-ray spin pulse of V1062 Tau we require dense partial absorbers varying over spin phase. At spin minimum the absorber is sufficiently dense as to be completely opaque. These results are consistent with absorption in the accretion curtains of material falling onto the magnetic poles. However, owing to ambiguity in the model fits, we cannot determine whether, in addition, parts of the accretion regions are passing over the white-dwarf limb. The need to average over substantial ranges of spin-phase, and the complexity of the models when both absorption and the normalisations of multi-temperature plasmas are allowed to change, means that unique solutions are not obtained. In reality, we should also include a reflection continuum, adding further phase-varying parameters and making the ambiguity worse.

Thus, even with combined ASCA and RXTE data, the complexities of apparently simple sinusoidal pulsations are such that spectral analysis is a poor diagnostic of the accretion flow, beyond the statement that dense $\left(>10^{23} \mathrm{~cm}^{-2}\right)$, phase-variable absorption is the primary factor.

\section{References}

Aizu, K. 1973, Prog. Theor. Phys., 49, 1184

Allan, A., Hellier, C., \& Beardmore, A. P. 1998, MNRAS, 295, 167

Bradt, H. V., Rothschild, R. E., \& Swank, J. H. 1993, A\&AS, 97,355

de Martino, D., Matt, G., Mukai, K., et al. 2001, A\&A, 377, 499

Kim, Y., \& Beuermann, K. 1995, A\&A, 298, 165

Hellier, C. 1991, MNRAS, 251, 693

Hellier, C. 1997, MNRAS, 291, 71

Hellier, C. 1999, in Annapolis workshop on magnetic cataclysmic variables, ed. C. Hellier, \& K. Mukai, ASP Conf. Ser., 157, 1

Hellier, C., Cropper, M., \& Mason, K. O. 1991, MNRAS, 248, 233

Hellier, C., Mukai, K., Ishida, M., \& Fujimoto, R. 1996, MNRAS, 280, 877

Hellier, C., Beardmore, A. P., \& Buckley, D. A. H. 1998, MNRAS, 299, 851

Mukai, K. 1999, in Annapolis workshop on magnetic cataclysmic variables, ed. C. Hellier, \& K. Mukai, ASP Conf. Ser., 157, 33

Patterson, J. 1994, PASP, 106, 209

Ramsay, G. 2000, MNRAS, 314, 403

Remillard, R. A., Bradt, H. V., Brissenden, R. J. V., et al. 1994, ApJ, 428, 785

Rosen, S. R., Mason, K. O., \& Cordova, F. A. 1988, MNRAS, 231, 549

Singh, J., \& Swank, J. 1993, MNRAS, 262, 1000

Tanaka, Y., Inoue, H., \& Holt, S. S. 1994, PASJ, 46, L37

Yaqoob, T. 2000, ASCA GOF Calibration Memo, ASCA-CAL00-06-02 